\title{
Assessment of Entrance Skin Dose and Image Quality of Chest $X$-Rays in Two University Teaching Hospitals, North East Nigeria
}

\author{
Dlama Joseph ${ }^{[1]}$ Joseph Igashi $^{[2]}$, Samuel Shem ${ }^{[3]}$, Peter Eshiet ${ }^{[4]}$, Dlama \\ Yabwa $^{[1]}$ Geofrey luntsi ${ }^{[1]}$,Abubakar Mundi ${ }^{[5]}$, Kpaku Goriya ${ }^{[6]}$ Gloria Joseph ${ }^{[7]}$ \\ ${ }^{1}$ Radiology Department, Abubakar Tafawa Balewa University Teaching Hospital Bauchi, P.M.B 1007 Bauchi \\ State, Nigeria. PIN Number 740001 \\ ${ }^{2}$ Ahmadu Bello University Teaching Hospital Zaria, Nigeria \\ ${ }^{3}$ Department of Radiography and Radiological Sciences, Nnamdi Azikiwe University Awka, Anambra \\ State, Nigeria \\ ${ }^{4}$ Radiology Department Federal Medical Center Keffi, Nasarawa State, Nigeria \\ ${ }^{5}$ Nasarawa State University Keffi, Nigeria \\ ${ }^{6}$ Nasarawa State University Keffi, Nigeria. \\ ${ }^{7}$ Gloria Joseph Abubakar Tafawa Balewa University Teaching Hospital Bauchi
}

\begin{abstract}
The need for radiation dose assessment of patients during diagnostic chest $x$-ray examination is considered in this study. Radiation exposure received by patients during routine $x$-rays is known to increase the risk of malignancy these was the major problem that necessitated this study. This study was carried out in two university teaching hospitals in Northeastern Nigeria. The aim and objective of this work was to assess the Entrance Skin Dose delivered to patients during routine chest $x$-ray examination and to evaluate the Image quality of the radiographs produced. Sixty thermoluminiscent dosimeters were placed on the patients during chest $x$-ray exposure to estimate the entrance skin dose received by each patient during exposure.The thermoluminescent dosimeters were analyzed at the Center for Energy Research and Training Zaria. Committee for European Guidelines 1999 criteria was used to analyze the image quality. Findings show that the dose obtained in the two hospitals were $0.50 \mathrm{mGy}$ and $0.54 \mathrm{mGy}$ respectively.The results obtained were high compared to other established work. The image quality criteria score was good with a score of $60 \%$ and above. Conclusion showed that there is need to optimize service in the two teaching hospitals and most hospitals in Nigeria.
\end{abstract}

Key Words:[ Radiation, Dose, Thermoluminiscent, Dosimeter, $X$-ray]

\subsection{Background of the Study}

\section{Introduction}

$\mathrm{X}$-ray is the most frequently used ionizing radiation for diagnostic imaging and it plays a significant role in effective health care delivery both in developed and developing countries ${ }^{[1,2]}$. X-ray is said to be the major contributor to the collective effective dose of the general public (personnel and patient). The need for radiation dose assessment of the patient during diagnostic x-ray examinations has been highlighted by increasing knowledge of hazard of ionizing radiation ${ }^{[3,5,20]}$. Because of the deleterious effects of $x$-rays, it is necessary to protect patients undergoing diagnostic and therapeutic procedures. The aim of any diagnostic $x$-ray examination is to produce images of sufficient and optimum quality. However, a good quality radiograph is not the one that is most appealing to the eye but, that in which sufficient details can be easily elicited.

In keeping radiation dose to patients to a minimum in hospitals, it is useful to be able to estimate prior to medical examination the dose to patients as a function of radiographic exposure parameters ${ }^{[6,7,9]}$. Radiation dose to patients from diagnostic $\mathrm{x}$-ray machine assures a simple functional dependence on radiographic exposure parameters of $\mathrm{kVp}, \mathrm{mAs}$, SSD, filtration and thickness ${ }^{[8,9]}$.

Monitoring of patients during the examination has been a major way of assessing radiation dose received in diagnostic and therapeutic radiography [10]. For the purpose of optimization in radiation protection, dose delivered to patients during diagnosis is studied with assessment of image quality ${ }^{[12,13]}$. This is a common practice in many parts of the world who present with clinical cases requiring $\mathrm{x}$-ray examination which are often times not properly done ${ }^{[14,27]}$. This is largely due to lack of facilities and suitable qualified personnel, as a result, there is no sufficient information about patient's radiation dose.

Patient's dose has often been described by the entrance skin dose (ESD) as measured in the centre of the x-ray beam. Because of the simplicity of its measurement, ESD is considered widely as the index to be assessed and monitored. ESD is measured directly using thermoluminiscent dosimeter (TLD) placed on the skin of the patient or indirectly from measurements of dose area product using a large area transmission ionization 
chamber (TIC) placed between the patient and the x-ray tube. The use of TLD technique requires prolonged annealing and reading process, furthermore, the use of TLD technique requires special equipments and thorough calibration facilities which may not be available in most x-ray departments.

On the other hand TIC method does not provide direct measurement of skin dose and mathematical equations which are needed to convert TIC reading into skin dose. Because of the limitation associated with both TLD and TIC several mathematical equations have been suggested to relate skin dose to the used exposure factor such as the applied mAs, surface to skin distance (SSD), filtration ,field size, output and the applied kVp. This equation provides an easy and more practical means of estimating skin dose even before exposure. Despite the attractive nature of the calculation methods of patient dose, one should make sure that the used $\mathrm{x}$-ray equipment has an adequate $\mathrm{QC}$ protocol that ensures the accuracy of the measured factors ${ }^{[16,17,20]}$.

Although, ESD may be sufficient for quality control measurements where the stability of the x-ray equipment is often of concern, the entrance dose is not sufficient for comparison or evaluation of actual patient dose and associated risk ${ }^{[16,25]}$.

The objective of this study is to estimate and assess ESD and image quality of chest $\mathrm{x}$-ray examinations and to use it as a standard in determining an easier method in assessing reference dose compliance criteria of patient's radiation exposure to medical $\mathrm{x}$-rays in the hospitals within this region.

\section{Materials And Methods}

The materials used for this study includes x-ray machine,TLD-100 dosimeter and patients who come for routine chest $\mathrm{x}$-ray examination. Characteristics of $\mathrm{x}$-ray machine is extracted from the available machine specific data such as type, model, waveform, filtration, year of manufacture, equipment age, generator type, dose reproducibility were recorded . X-ray machine setting such as $\mathrm{kVp}$ and $\mathrm{mAs}$ values for each examination were taken directly from the control panel. ESD of 60 patients has been considered in this study. The dose survey was conducted between September 2014 to November 2014. Adult patients of both sexes were randomly selected in ATBUTH Bauchi, and UMTH North Eastern Nigeria. Critically ill patients and pediatric patients were excluded from this procedure. After due consent from the patients and ethical committee of both hospitals, the patients, routine chest PA or AP procedures were examined for dose assessment. For each patient, the following parameters were recorded: sex, age, weight and height and Body Mass Index (BMI).

This information about the patients, together with body mass index (BMI) derived from weight $(\boldsymbol{k g})$ /(height $(\boldsymbol{m}))^{2}$, which is a useful classification scheme for size and shape of a person (Gibson, 1990).

The weight and height of each patient were measured using ZT-160 WHO hospital scale with both weight and height scale. Maximum capacity for the weight scale is $120 \mathrm{~kg}$ and with error of $\pm 1.2 \mathrm{~kg}$ for weight below $60 \mathrm{~kg}$ and $\pm 2.0 \mathrm{~kg}$ for weight above $60 \mathrm{~kg}$. Entrance skin dose was estimated using TLD readings to give the entrance air kerma. Entrance skin dose represents the radiation intensity as the x-ray beam impinges on the surface of the skin. The skin dose, at the very surface of the skin where no attenuation has occurred, it is computed as

$E S D=E A K X B S F$

Equation 2.1

Where $\mathrm{EAK}=$ Entrance air kerma, $\mathrm{BSF}=$ Back scatter factor, $\mathrm{ESD}=$ Entrance skin dose $\mathrm{x}$-ray machines The machine settings were $\mathrm{kVp}$ readings of 0 to 300 and mAs readings of 1 to 500. Entrance skin dose, ESD is the absorbed dose to air on the X-ray beam axis at the patient skin where the X-ray beam enters the patient. But to compute the ESD, we first of all have to obtain an incident absorbed dose to air (IDair) which is defined as the absorbed dose to air on the X-ray beam axis at the focus -to-skin distance (FSD).These values were obtained directly from the TLD. Thus, the ESD is related to the incident absorbed dose by backscatter factor (BSF) which is the ratio of the incident dose in tissue to the incident dose in air. ESD of each patient were calculated by multiplying the patient's air kerma by a back scatter factor of 1.06 as suggested in the European guidelines (EC, 1996).

Therefore, according to Tung and Tsai et al., 1996 and Shrimptonet al., (1986).

$$
E S D=I D \text { air } \times B S F
$$

This equation was used to compute the ESD for each patient. The values for the ESD obtained were analyzed using Statistical Package for social sciences (SPSS) version 16.0. Maximum dose, minimum dose $1^{\text {st }}$ quartile and $3^{\text {rd }}$ quartile values were obtained. Image quality was obtained by strict adherence to the guidelines suggested by European committee, 1996.

Image quality of chest radiography seeks to establish the level of compliance with international standards using Committee for the European Community (CEC) guidelines for image quality. This will also provide a basis for optimization of the chest radiographic procedure in the hospitals. Assessment of image quality in this study was carried out in each hospital by two experienced and certified Radiologist and Radiographers in other to ensure compliance with guidelines. The assessment was strictly and carefully done using the format in table 2.1. The image quality scoring was based on comparison of respective radiographs with 
a reference image selected from the archive which both assessors agreed shows full compliance with CEC guidelines. Each assessor worked independently, separately studying the radiographs from each of the hospitals.

No reference film was used for technical assessment criteria. However, the radiographers, working were asked to score the films from 1 to 3 with 3 being the highest score. Positioning was listed under the radiographic/technical criteria to enable computation of number of films that fail this assessment. Assessment method includes:

i. Poor/to be repeated

ii. Acceptable

iii. Good quality

For beam collimation, score 1 indicated radiograph which needs improvement and did not necessarily imply a repeat of the examination. A table for the assessment of criteria used for subjective scoring in this work is given below

\section{Presentation And Analysis Of Data}

Table 3.1: Personnel and radiographic equipment data used in the hospitals Machine parameters are presented as labeled on the machines in the hospitals.

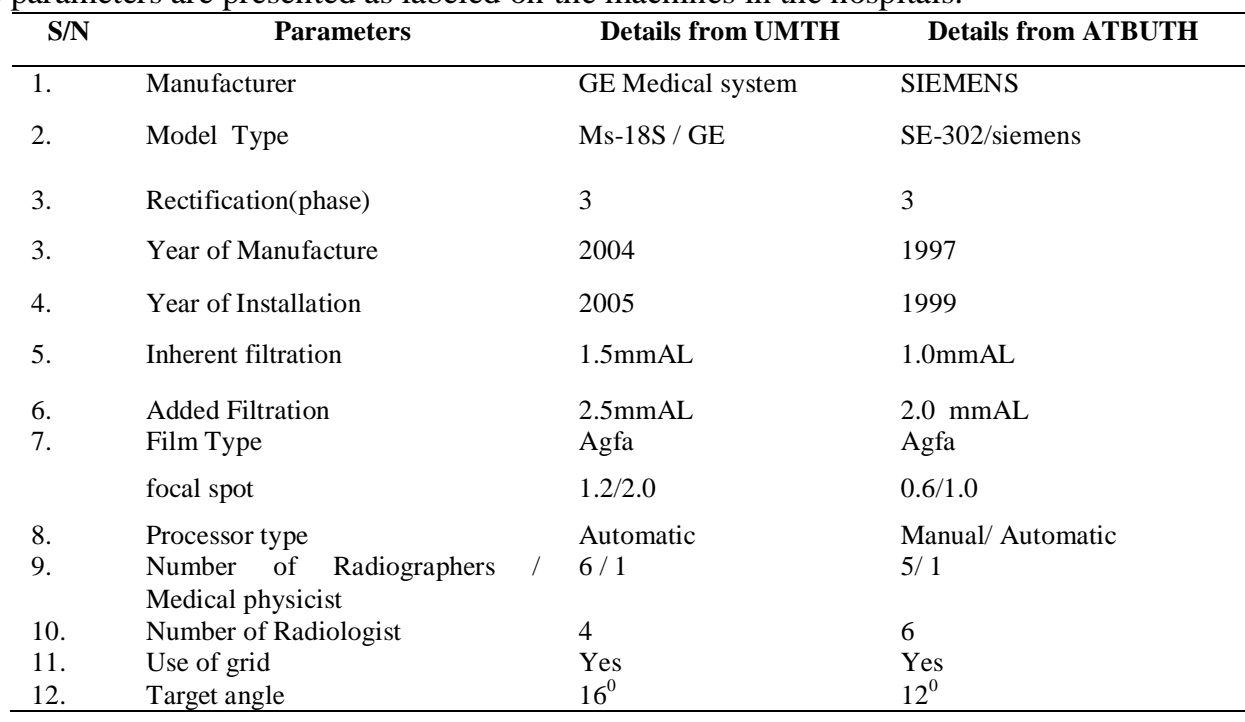

The table above shows that the machines in UMTH and ATBUTH are relatively new, the inherent filtration and added filtration are relatively the same. Both machines are gridded.

Table 3.2: Mean (range) of radiographic parameters used in X-ray centers.

\begin{tabular}{cllll}
\hline Examination & \multicolumn{1}{c}{ X-ray center } & \multicolumn{1}{c}{ kVp } & \multicolumn{1}{c}{ mAs } & FSD $(\mathbf{c m})$ \\
\hline Chest X-ray & ATBUTH & $68(60-70)$ & $16(12-20)$ & $135(120-154)$ \\
& UMTH & $71.5(60-82)$ & $18(6-26)$ & $130(120-155)$ \\
\hline
\end{tabular}

Table 3.2 shows that the mean $\mathrm{kVp}$ value of 68 and 71.5 for ATBUTH and UMTH respectively the variation may likely be as a result of different machine type, patient thickness and FFD used for various chest $\mathrm{X}$ rays. mAs value for UMTH is 18 while that of ATBUTH is 16 .

Table 3.3: $\quad$ Sex distribution, mean (range) of age, weight and BMI of patients

\begin{tabular}{lll}
\hline \multicolumn{1}{c}{ X-ray center } & \multicolumn{1}{c}{ UMTH } & \multicolumn{1}{c}{ ATBUTH } \\
\hline No. of patients & \multicolumn{1}{c}{30} & 30 \\
Sex & 15 males, 15 females & 15 males, 15 females \\
Age (year) & $36(18-50)$ & $48(19-80)$ \\
Weight $(\mathrm{kg})$ & $50(38-80)$ & $59(40-82)$ \\
Height $(\mathrm{m})$ & $1.48(1.3-1.9)$ & $1.56(1.3-1.8)$ \\
Thickness $(\mathrm{cm})$ & $15(10-35)$ & $16.9(12-23)$ \\
BMI $\left(\mathrm{kg} / \mathrm{m}^{2}\right)$ & $26(18-40)$ & $25(18-41)$ \\
\hline
\end{tabular}

Table 3.3 showed that the patients considered for this study were between the ages of 18 to 80 years of age with average age of 36 year and 48 year in UMTH and ATBUTH respectively. Patients thickness for both hospitals were closely related $15 \mathrm{~cm}$ and $16.9 \mathrm{~cm}$ for UMTH and ATBUTH respectively with Body Mass Index (BMI) of $26 \mathrm{~kg} / \mathrm{m}^{2}$ and $25 \mathrm{~kg} / \mathrm{m}^{2}$ for UMTH and ATBUTH . 
Table 3.4: Individual entrance skin dose (ESD) distribution for the two hospitals with their minimum and maximum values.

\begin{tabular}{lcccccc}
\hline Hospital & $\begin{array}{c}\text { Min } \\
(\mathbf{m G y})\end{array}$ & $\begin{array}{c}\mathbf{1}^{\text {st }} \text { quartile } \\
\mathbf{m G y}\end{array}$ & $\begin{array}{c}\text { Mean } \\
\mathbf{m G y}\end{array}$ & Median & $\begin{array}{c}\mathbf{3}^{\text {rd }} \text { quartile } \\
\text { mGy }\end{array}$ & $\begin{array}{c}\text { Max } \\
\text { mGy }\end{array}$ \\
\hline ATBUTH & 0.01 & 0.33 & 0.50 & 0.47 & 0.70 & 0.99 \\
UMTH & 0.22 & 0.39 & 0.54 & 0.49 & 0.60 & 0.68 \\
\hline
\end{tabular}

Table 3.4 shows $1^{\text {st }}$ and $3^{\text {rd }}$ quartile values of $0.33 \mathrm{mGy}$ and $0.70 \mathrm{mGy}$ for ATBUTH and $0.39 \mathrm{mGy}$ and $0.68 \mathrm{mGy}$ for UMTH. Mean doses are $0.50 \mathrm{mGy}$ and $054 \mathrm{mGy}$ for ATBUTH and UMTH respectively

Table 3.5: $\quad$ Comparison of radiographic parameters used in this work and other published work in four

\begin{tabular}{|c|c|c|c|c|c|c|}
\hline \multirow{2}{*}{$\begin{array}{l}\text { Projection / } \\
\text { Parameters }\end{array}$} & \multirow{2}{*}{$\begin{array}{l}\text { TaiwanTung et } \\
\text { al,. } 2001\end{array}$} & \multirow{2}{*}{$\begin{array}{c}\text { Malaysia Kia et } \\
\text { al,. 1998 }\end{array}$} & \multirow{2}{*}{$\begin{array}{c}\text { Portugal Serro } \\
\text { et al,. } 1992\end{array}$} & \multirow{2}{*}{$\begin{array}{c}\text { UK NRPB } \\
1996\end{array}$} & \multicolumn{2}{|c|}{ This Work } \\
\hline & & & & & ATBUTH & UMTH \\
\hline Chest PA & & & & & & \\
\hline $\mathrm{kVp}$ & 77 & 79 & 76 & 76 & 68 & 71.5 \\
\hline $\mathrm{mAs}$ & 16 & 9 & 12 & 8 & 16 & 18 \\
\hline
\end{tabular}

From Table 3.5 above thekVps of others established studies has been found to be higher than this work (ATBUTH 68 and UMTH 71.5). The mAs ofTaiwan and this study are closely related. Thatof malaysia and UK (9 and 8) are less.

Table 3.6: $\quad$ Comparison of the mean value of ESD from four countries and this work.

\begin{tabular}{ccccccc}
\hline Projection & $\begin{array}{c}\text { Taiwan Tung et } \\
\text { al. 2001 }\end{array}$ & $\begin{array}{c}\text { Malaysia Kia et } \\
\text { al 1998 }\end{array}$ & $\begin{array}{c}\text { Portugal Serro } \\
\text { et al. 1992 }\end{array}$ & $\begin{array}{c}\text { UK NRPB } \\
\mathbf{1 9 9 6}\end{array}$ & \multicolumn{2}{c}{ This Work } \\
ATBUTH & UMTH \\
\hline Chest PA & 0.52 & 0.28 & 0.31 & 0.16 & 0.50 & 0.54 \\
\hline
\end{tabular}

Table 3.6 shows that the ESD of Taiwan Tung et al,.and this study $(0.52 \mathrm{mGy}, 0.50 \mathrm{mGy}$ and $0.54 \mathrm{mGy})$ are higher compared to the study in Malaysia, Portugal and UK $0.28 \mathrm{mGy}, 0.31 \mathrm{mGy}$ and $0.16 \mathrm{mGy}$ ) respectively.

Table 3.7: $\quad$ Assessment of the $\%$ of Image Quality Criteria in this work.

This table shows image quality criteria as obtained from various radiographic images obtained from the two hospitals.

\begin{tabular}{|c|c|c|c|c|c|c|c|c|c|c|}
\hline $\begin{array}{c}\text { Image } \\
\text { criteria } \\
\text { code }\end{array}$ & {$[\mathbf{A}]$} & [B] & {$[\mathrm{C}]$} & [D] & {$[\mathbf{E}]$} & {$[\mathbf{F}]$} & [G] & {$[\mathbf{H}]$} & {$[\mathbf{J}]$} & {$[\mathbf{K}]$} \\
\hline $\begin{array}{l}83.6 \% \\
\text { ATBUTH }\end{array}$ & 26 & 20 & 27 & 24 & 26 & 29 & 20 & 28 & 26 & 25 \\
\hline $\begin{array}{l}87.3 \% \\
\text { UMTH }\end{array}$ & 26 & 22 & 28 & 25 & 27 & 29 & 24 & 27 & 26 & 28 \\
\hline
\end{tabular}

Table 3.7 revealed that the image quality criteria shows good compliance with percentage score of $83.6 \%$ and $87.3 \%$ for ATBUTH and UMTH respectively.

Table 3.8: $\quad$ Total score of radiographic / technical quality criteria and $\%$ mean of number of film analysis score per hospital in this study

\begin{tabular}{lcccccc}
\hline \multicolumn{1}{c}{ Criteria } & \multicolumn{3}{c}{ ATBUTH $(\mathbf{n}=\mathbf{3 0})$ Score } & \multicolumn{4}{c}{ UMTH (n= 30) } \\
\hline Optical density & {$[\mathbf{1}]$} & {$[\mathbf{2}]$} & {$[\mathbf{3}]$} & {$[\mathbf{1}]$} & {$[\mathbf{2}]$} & $\mathbf{3}$ \\
Collimation & $2(6.6 \%)$ & $4(13 \%)$ & $24(80 \%)$ & $1(3.3 \%)$ & $3(10 \%)$ & $26(86 \%)$ \\
Positioning & $5(16 \%)$ & $7(24 \%)$ & $18(60)$ & $2(2.6 \%)$ & $5(16 \%)$ & $23(77 \%)$ \\
Marker / ID & $3(10 \%)$ & $6(20 \%)$ & $21(70 \%)$ & $2(2.6 \%)$ & $3(10 \%)$ & $25(83 \%)$ \\
Processing & $1(3.3 \%)$ & $2(6.6 \%)$ & $27(90 \%)$ & $1(3.3 \%)$ & $2(6.6 \%)$ & $28(93 \%)$ \\
\hline
\end{tabular}

From Table 3.8 above, there is good quality for Radiographic and technical parameters in both hospitals with greater $(>) 60 \%$ score for each criteria assessed. 


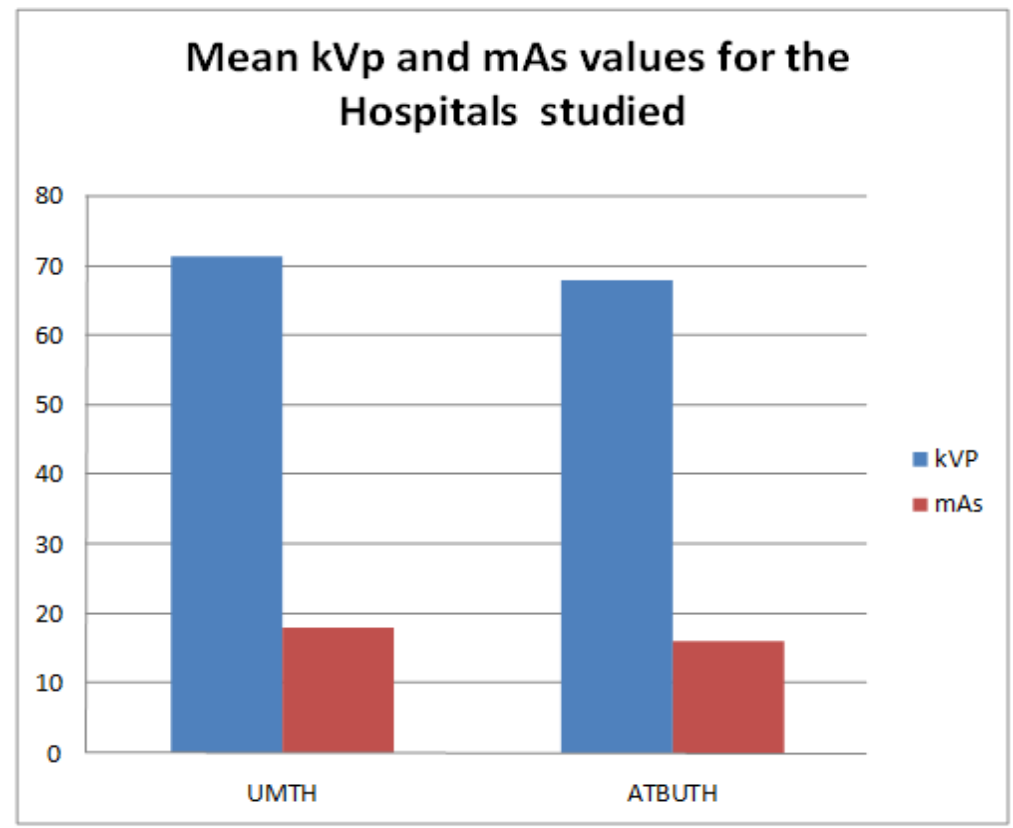

Figure 3.1:Comparison of the $\mathrm{kVp}$ and $\mathrm{mAs}$ obtained in ATBUTH and UMTH.

From figure 4.1 UMTH has mean $\mathrm{kVp}$ and mAs values of 71.5 and 18 while ATBUTH has 68 and 16 respectively.

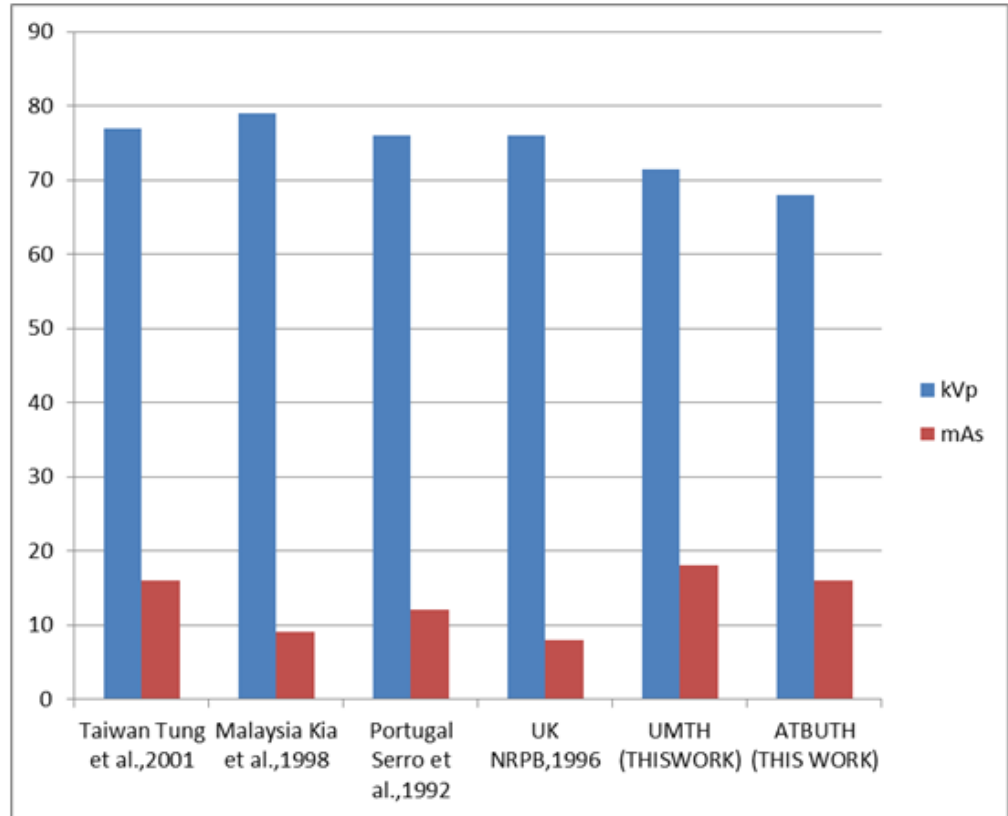

Figure 4.2: Comparison of mean values of radiographic parameters used in this work and other surveys in four countries.

The figure above shows that the $\mathrm{kVp}$ of other established studies has been found to be higher than that of this work (ATBUTH 68 and UMTH 71.5). The mAs of Taiwan and these studies are closely related. That of Malaysia and UK (9 and 8) are less. 


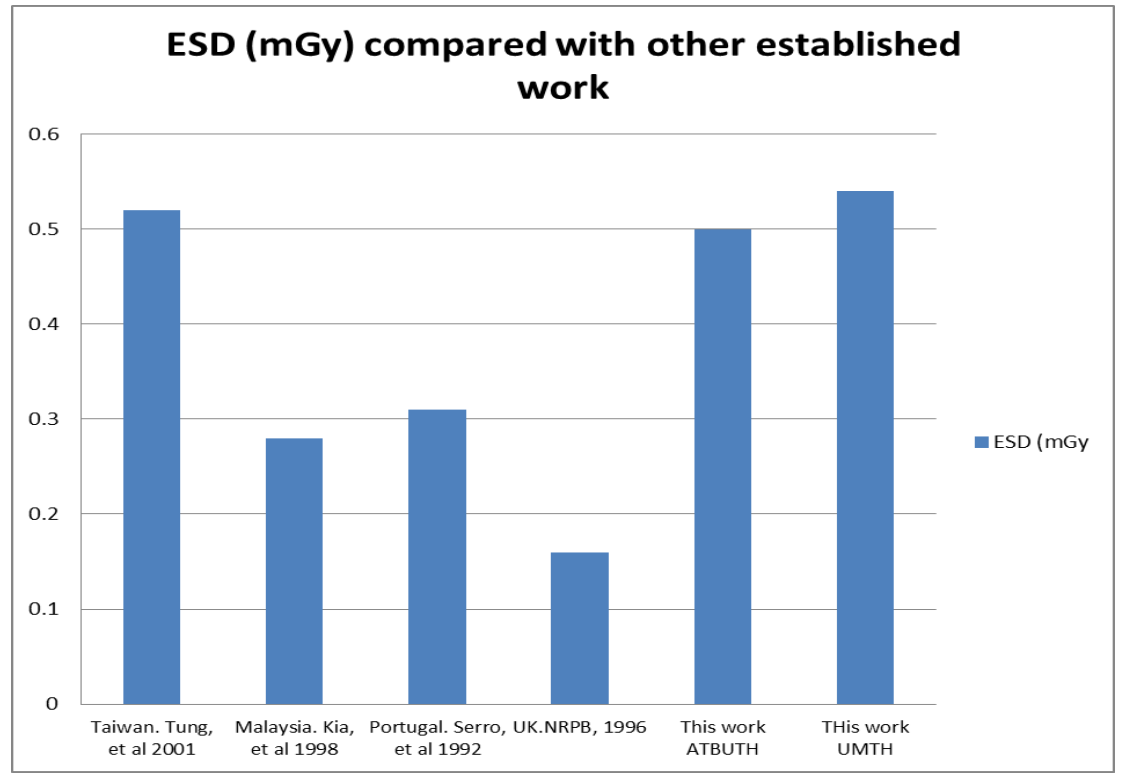

Figure 3.3: $\quad$ Comparison of the mean value of ESD from other four countries and this work.

From the figure above the ESD of Taiwan Tung et al., and this study $(0.52 \mathrm{mGy}, 0.50 \mathrm{mGy}$ and $0.54 \mathrm{mGy})$ are higher compared to the study in Malaysia, Portugal and UK $(0.28 \mathrm{mGy}, 0.31 \mathrm{mGy}$ and $0.16 \mathrm{mGy}$ respectively).

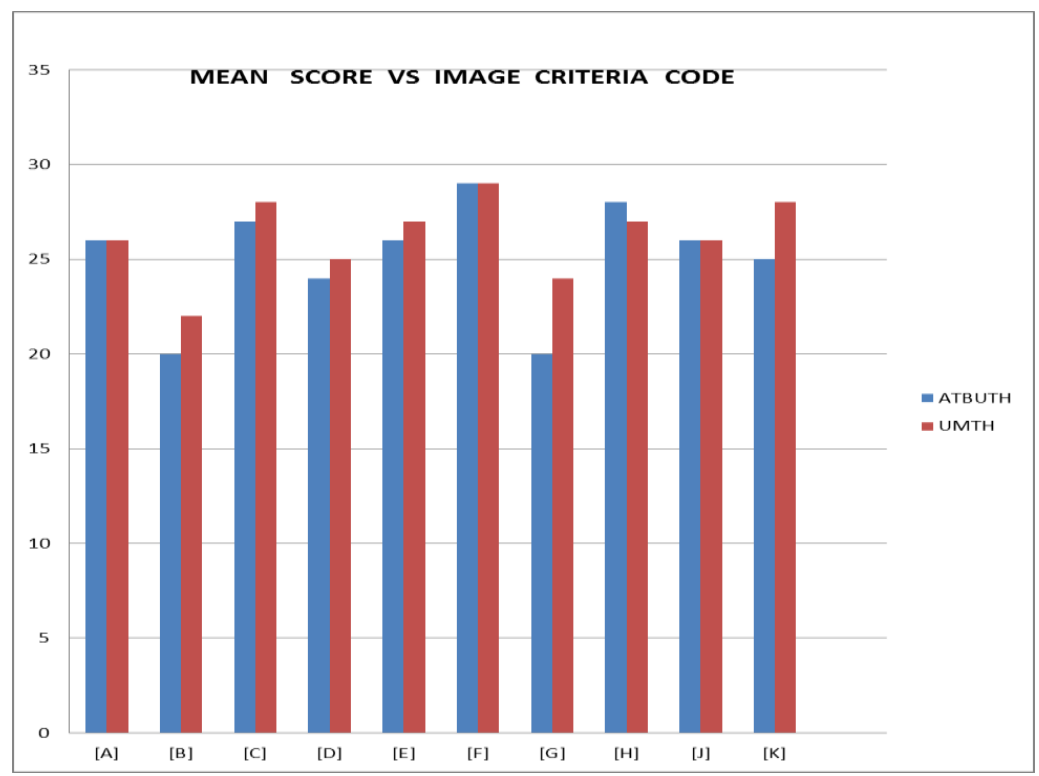

Figure 3.4: Mean observer score versus image criterion

From the figure above, there is good compliance with image quality criteria in both hospitals studied with 20 films and above out of thirty(30) films having good score. 


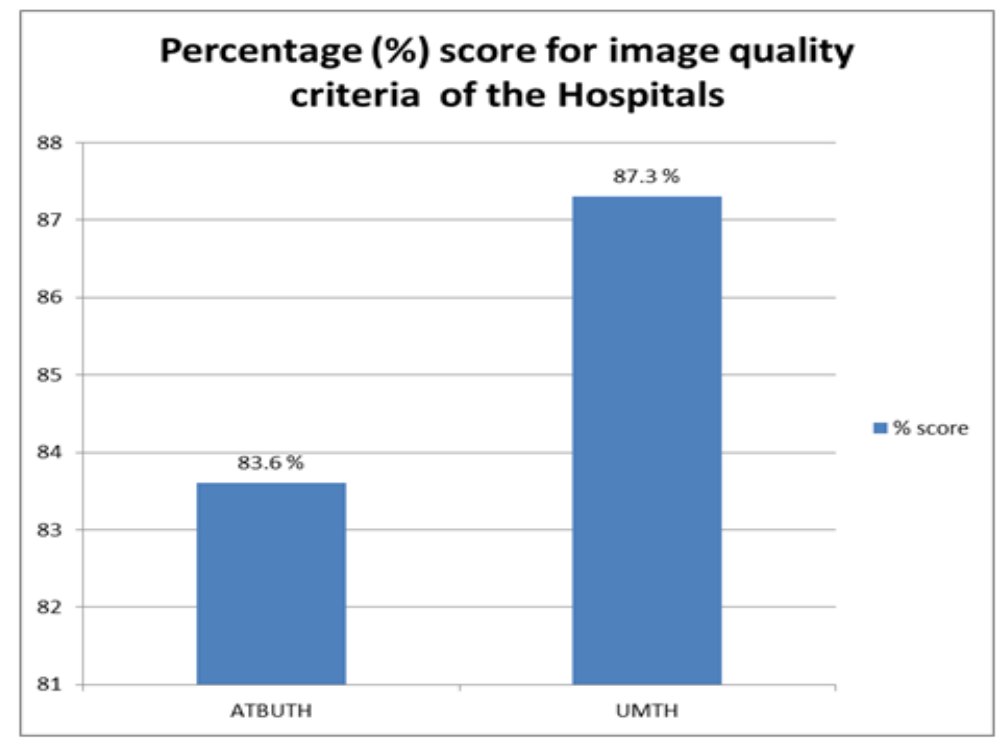

Figure 3.5: Percentage score for image quality criteria

Figure above shows that shows percentage score for image quality criteria for ATBUTH (83.6\%) and $\operatorname{UMTH}(87.3 \%)$.

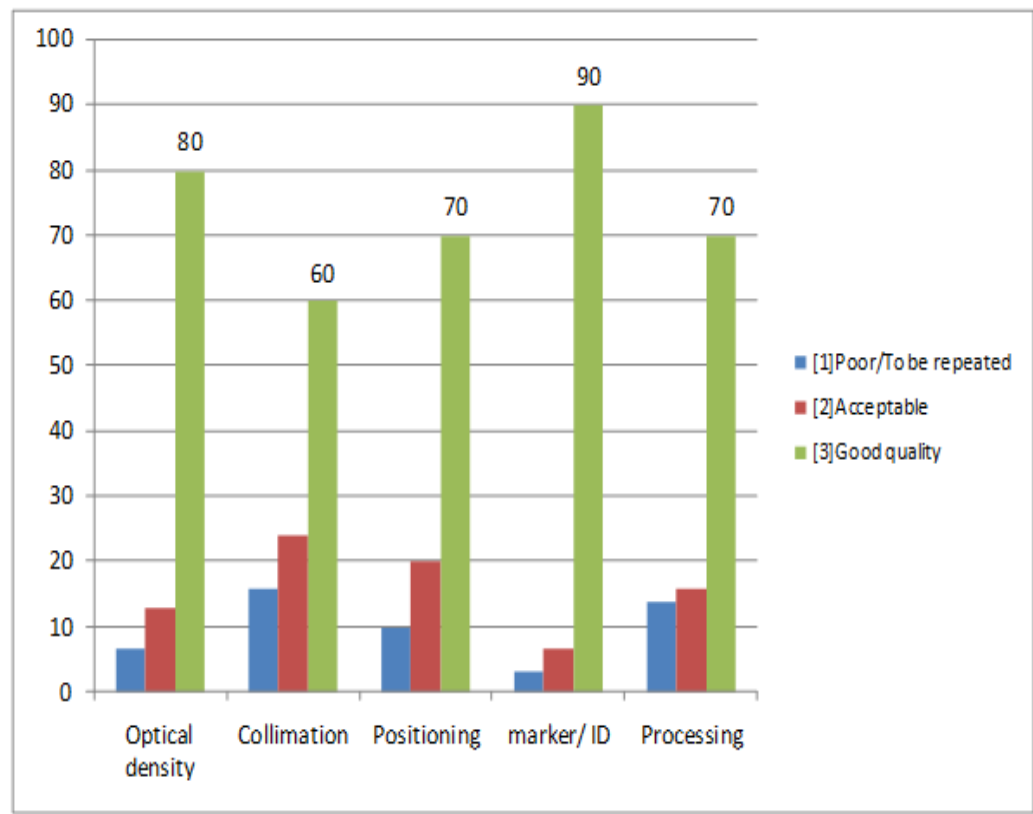

Figure 4.6: Percentage scoreof radiographic/ technical quality in ATBUTH

From the figure above the percentage score for the radiographic and technical qualities in ATBUTH were good with the least score of $60 \%$ score for good quality radiograph, the rest were above $60 \%$. 
Figure 3.7: Percentage score of the total number of radiographic/technical quality

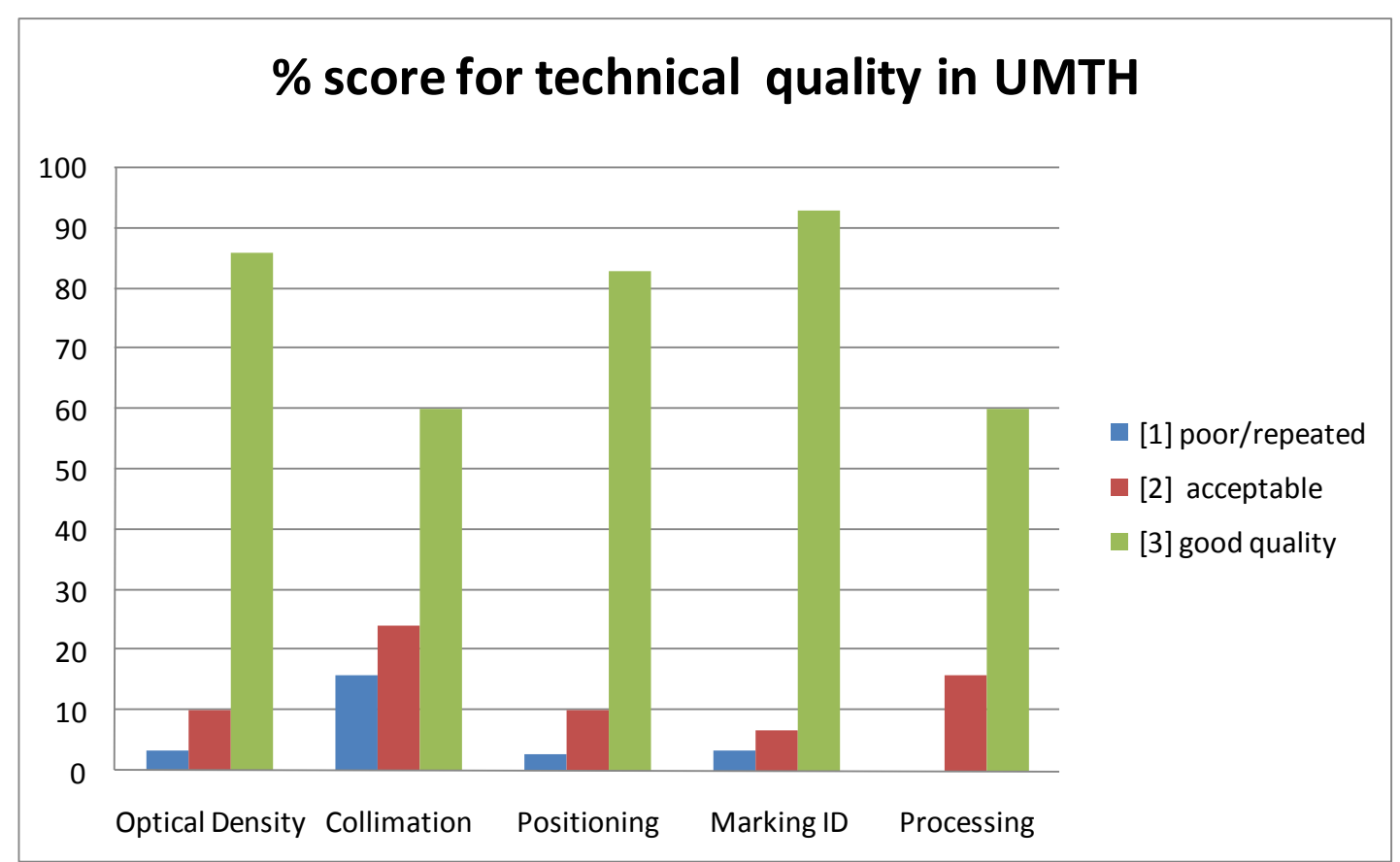

From the figure above the percentage score for the radiographic and technical qualities for the images in UMTH were good with over $60 \%$ score for good quality radiograph.

\section{Discussion}

The result of this study provides a baseline for local diagnostic reference levels(LDRLs) in the two hospitals considered in this study.

The variation in patient dose for the same type of x-ray examination carried out on similar patients in the two hospitals compared to other established work suggests that significant reductions in the dose from this exposures would be possible without adversely affecting image quality, such reductions should always be pursued with radiation safety in view.

A common position among the hospitals in Nigeria is the lack of regular patient dose monitoring and quality control in diagnostic radiology. A major reason for this is the cost of running a standard radiation protection and quality assurance facility. This is in consonance with the study by Egbe et al., (2008).

A total of 60 patients from two Teaching hospitals in North eastern region of Nigeria were included in this study. The summary of the technical parameters (tube voltage and milli Ampere seconds) used for the PA chest x-ray for the patients in the two centers are given in table 3.2. Results from table 3.2 show that the mean value for $\mathrm{kVp}$ and mAs of ATBUTH( 68 and 16) is lower compared to that of UMTH (71.5 and 18). Comparison of these parameters with those obtained in similar surveys(Tung et al.(2001), Kia et al (1998), Serroet al., (1992) and NRPB, (1992) are presented in Table 3.5.

Table 3.1 above shows that the machines in UMTH and ATBUTH are relatively new, the inherent filtration and added filtration are relatively the same. Both machines are gridded.

Table(3.5) shows that while the mean $\mathrm{kVp}$ values obtained in other studies are between 70 to $80 \mathrm{kVp}$ that of UMTH is 68 though not much variation. The mean mAs of UMTH (18mAs) is higher compared to other published work. This study disagree with the findings of Agba et al.,(2002) but concur with the findings of Egbe et al., (2008). Reason for statistically significant differences are recorded between calculated and measured doses for the hospitals. This could also be attributed to larger variability in generator output, itself as a result of the unavailability of quality control programs in the studied hospitals. Higher mAs recorded in UMTH may be as a result of poor reproducibility status of the x-ray machine. This study also showed that body habitus, thickness of patient's chest and focus to film distance applied tends to affect exposure parameters and image quality.

Table 3.3 gives the ESD summary of PA chest obtained in the two Teaching hospitals while Table 3.5 compares the finding with those obtained in four different published works. The range of ESD in the tables shows that in a given center the range of factor(maximum entrance dose divided by minimum entrance dose) is mostly between 1 and 2 but there are variations as obtained from the two teaching hospitals in this work. These variations may be closely attributed to the fact that patient's examination in this center is less optimized. 
During medical examinations, it is sometimes necessary to use the grid for follow-up examinations of robust patients' especially those undergoing lateral chest X-ray. As recommended, a radiation worker should not be exposed to more than $20 \mathrm{mSv}$ over a consecutive period of 5 years.

However,for the patients undergoing X-ray, examination is justified and the radiation dose to the patients is kept as low as reasonably achievable (ALARA principle), the mean dose value $(0.54$ and $0.50 \mathrm{mGy})$ for ATBUTH and UMTH in this research study was quite higher compared to other established work;reasons most likely due to patients' thickness and filtration of the machine.

A comparison of doses from this work and with those from the work of Tung et al, (2001), Kia et al.,(1998), Serro et al., (1992) and NRPB, (1996) is presented in Table 3.5. This work confirmed that patients who underwent PA chest $\mathrm{x}$-ray examination receive higher radiation dose compared to other international published work by Kia et al.,(1998), Serro et al.,(1992) and NRPB (1996) but almost close to that obtained by Tung et al.,(2001). The reason behind the higher doses may be attributed to differences in technical parameters used. The high dose could also be due to patient size or clinical complexity, suboptimal usage of equipment or equipment problems generally because of the paucity of regular quality control and radiation protection program. This variation clearly shows the need for patient dose reduction in the two centers used in this work. However, patient dose reduction has been a long standing issue Sharifat et al., (2009).

This work unveils that despite all efforts to reduce radiation as low as reasonably achievable taking into consideration economic and social factors, there still exist centers where patient's doses can be very high most especially in developing countries.

Table 3.7 shows the image quality assessment criteria. Both radiographic/ technical quality and image quality using CEC criteria results in this study show a good performance with radiographic quality of $83.6 \%$ and 87.3\% in ATBUTH and UMTH respectively compared to the work done by Egbe et al., (2008) (53.7\%). However, the result of the radiographic quality of the two hospitals considered in this study show wide variation probably due to increasing number of experienced Radiographer and Radiologist.

A good score of $83.6 \%$ and $87.3 \%$ (ATBUTH and UMTH) was obtained for all images studied with respect to CEC criteria. Europian Commission, (1996). This value implies that the value classified as poor is by far lower than that reported by Satoet al., (1995). The mediastinal, retrocardial and spinal area (as seen through the heart) of the chest images recorded the highest marging in the image quality criteria. This agrees with the result of Egbe et al., (2009). The image score obtained in this study may be due to the fact that both majority of the patients were of average to below average size and the use of Radiographers in imaging procedures.

A review of the films that failed the technical quality assessment for ATBUTH and UMTH respectively showed that $2(6.6 \%)$ and $1(3.3 \%)$ had poor optical density, $6(20 \%)$ and $(2 \%)$ had improper collimation, 3(10\%) and $2(2.6 \%)$ had positioning faults. About $1(3.3 \%)$ and $1(3.3 \%)$ of the total number of films were not properly identified of had a wrong anatomical marking and $8(26 \%)$ and $2(2.6 \%)$ had positioning problems however, are all correctable problems. A review and adoption of an optimization protocol would help in reducing the identified shortfalls in image quality and reduce dose. The development and consistent use of quality assurance service is therefore suggested, in this way possible changes in radiographic technique, to use high tube potentials and short exposure times, can be determined for each center and for respective examinations.

\section{Summary of the Study Findings}

1. The maximum ESD (Entrance Skin Dose) in ATBUTH and UMTH were $0.68 \mathrm{mGy}$ and $0.99 \mathrm{mGy}$ respectively.

2. The minimum ESD (Entrance Skin Dose) in ATBUTH and UMTH were $0.01 \mathrm{mGy}$ and $0.22 \mathrm{mGy}$ respectively

3. The mean ESD of ATBUTH and UMTH were $0.50 \mathrm{mGy}$ and $0.54 \mathrm{mGy}$ which were higher than the recommended dose of $0.4 \mathrm{mGy}$ by ICRP (1992),(NRPB,2002).

4. There is good compliance with image quality criteria in both hospitals with percentage score of $83.6 \%$ in ATBUTH and $87.3 \%$ in UMTH.

5. Technical and radiographic quality assessment was goodin both hospitals with percentage score of $60 \%$ and above in both hospitals.

\section{Conclusion}

In this study, entrance skin doses from two teaching hospitals in North eastern part of Nigeria were presented. The mean entrance skin dose at ATBUTH and UMTH were higher compared to published work and recommended dose of $0.4 \mathrm{mGy}$ by (ICRP,2001),(NRPB,2002) for patients undergoing PA chest $\mathrm{x}$-ray examination consistent with ALARA even at the same radiologic and geometric parameters. The mean entrance doses, obtained from measurements carried out on 60 patients were $0.50 \mathrm{mGy}$ and $0.54 \mathrm{mGy}$ for ATBUTH and UMTH respectively. The high doses are a pointer and further indications that dose delivered to patients' are not as low as reasonably achievable in many Nigerian hospitals. These also shows that despite all efforts towards 
reduction of patients doses, there still exist center's where patients' doses are still high especially in developing countries. The major contributor to the high dose in this work is attributed to patient size, clinical complexity, suboptimal usage of equipment or equipment problems generally because of the paucity of regular quality control and radiation protection program. However, image quality and technical quality assessment were good.

\section{Recommendations}

i. This research work shows that there is a need to optimize operations in ATBUTH and UMTH and probably in Nigeria at large especially for chest $\mathrm{x}$-ray examination which is the commonest examination.

ii. The optimization step may start with the regulatory body mandating radiographers and radiologist and medical physicist to take part in various refresher and update course for them to be aware of the recent developments on how to properly and effectively select technical parameters that will not affect or compromise image quality

iii. The hospitals should implement a standing and functional radiation safety committee; appoint a radiation safety adviser and radiation safety officer that will be trained by the regulatory body on Radiation safety. The essence of enacting this committee is to saddle them with the responsibility of monitoring the staff and ensuring Radiation safety in the hospital.

iv. There is also need for national survey so as to set a guidance dose for this examination so that hospitals can always compare their dose and take remedial action without affecting image quality.

v. A culture of regular dose measurement, film reject analysis, and image quality assessment should be inculcated as recommended by IAEA need to become part of diagnostic radiology procedure.

\section{References}

[1] Agba E.H, Fiase J.O, Agada S.A, and Thomas Dickson (2002)Patients skin dose from diagnostic X-rays at Federal Medical Centre Markudi, Benue State. Zuma Journal of Pure and Applied Science Vol.4(i)p14-18.

[2] Babalola I.A, (2004) "in quest of light Quanta", University of Ibadan Inaugural lecture series.Vol 10.p25-30

[3] Birtch R, and Maeshall M. (1979) Computation of bremsstrahlung X-ray spectra and comparison with spectra measured with Ge(Li) detector. Phs. Med BiolVol 24: 505-517.

[4] Brennan PC. MC Donnell and Leanly D.O (2004) Increasing FFD Reduces Radiation Protection, Dosimetry, Oxford University Press Vol.108 no.3, p263-68.

[5] BushongSc: Radiography Science for Technologist Physics, Biology and Protection $5^{\text {th }}$ edition St. Louis, Mo: Mosby year book 1993.

[6] Christian Norquist (2011) Effectof Radiation on Humans. http://www.medicalnewstoday.com/articles/219615

[7] Curry T.S,Dowdey J.E, Marry R.C Christensen's Introduction to the Physics of Diagnostic Radiography $3^{\text {rd }}$ ed. lea and Fibiger, Philadelphia PA, 1984

[8] Draft of ICRP Committee 3 on Diagnostic Reference Levels in Medical Imaging.

[9] Edmonds IR (1984) Calculation of Patient Skin Dose from Diagnostic X-ray Procedure.British Journal of Radiology 57, 733-734

[10] Egbe N.O, Inyang S. O., Eduwem D. U., and Ama I. (2009) Doses and Image Quality for Chest Radiograph in Three Nigerian Hospitals.European Journal of Radiography 2009, 1, 30 - 36

[11] Euratom of 30 June 1997 on Health Protection of Individuals Against the Dangers of Ionizing Radiation in Relation to Medical Exposure.

[12] European Commission (1997); Council Directive 97/43

[13] European Commission, (1996) European Guidelines on Quality Criteria for Diagnostic Radiographic Images, EUR, 16260 EN, Luxembourg. Office for Official Publications of European Communities 1996.

[14] Gibson R. S. (1990); Principle of Nutritional Assessment. New York, Oxford University Press 1990: $178-82$

[15] Hanan, F, (2007) Assessment of Entrance Skin Dose and Effective Dose of Some Routine X-ray Examinations using Calculation Technique published m.sc theses online

[16] Harrison RM, Clayton C. B., Day M. J. Owen JP, and York M. (1993).A Survey of Radiation DosetoPatients in Five Common Diagnostic Examinations.British Journal of Radiology 66, 383 - 395

[17] Hart D, Jones D. J. and Wall B. F. NRDB Report R262.

[18] ICRP 2000 "Recommendation of International Commission on Radiological Protection" ICRP Publication64 Ann ICRP 199121 (1 3) Pergamum Press, Oxford UK.

[19] International Atomic Energy Agency: International Basic Safety Standards for Protection against Ionizing Radiation and for safety of radiation sources IAEA Safety Series No. 115-1, Vienna Austria 1994.

[20] Johnson and Brenan (2000). Reference Dose Levels is for Patients Undergoing Common Diagnostic X-rays Examination in Irish Hospitals. British Journal of Radiology 73. $396-402$

[21] Kia N.G, RasiahP,Wang H.B, Hambali A.S, Muthuvelli P and Lee H.P.(1998). Doses to patients in routine X-ray examinations in malaysia. British Journal of Radiology 71,654-660.

[22] National Radiological Protection Boards: Patient Dose Reduction in Diagnostic Radiology: Document of the NRPB (3) 1990.

[23] Nworgu, O.D., Bamidele, L.(2010) An evaluation of entrance surface doses from some common diagnostic x-rayexaminations in two Nigerian university teaching hospitals. West African journal of Radiologyvol 17(1) 11-17

[24] Ogunseyinde A.O, Adeniran Sam, Obed R I,Akinlade B I, Ogundare F.O, (2002) Comparison of entrance skin dose of some X-ray examinations with CEC reference doses. Rad protdosimetry98(2):231-4.

[25] Olarinoye I. O. and Sharifat I. A, (2008)A Protocol for setting dose reference level for medical Radiography inNigeria: A Review Bayero Journal of Pure and Applied Science Vol. 3 (1): 43-46.

[26] Olowookere C. J., Babalola I. A, Jibrin N. N. Obed I. R., Bamidele L. and Ajetumobi E. O.(2012). A Preliminary Radiation Dose Audit in some Nigerian Hospitals: a need for determination of Diagnostic Reference Level (NDRLs): Pacific Journal of Science and Technology Vol. 13. (1): $487-494$.

[27] Ragulla D.F, Eder H,(2005)Patient exposure in medical imaging in Europe. Radiation protection dosimetry. 114(1-3): 11-25. 
[28] Sato, O. Iwai, S. Tanaka, S. Vehara T, Sakamoto Y, Yoshzawa N, and Farihata S. (1995).Calculation ofEquivalent Dose and Effective Dose Conservation Coefficients for Photon from MeV to $10 \mathrm{GeV}$.Radiation Protection DosimetryNuclear Technology Publishing Japan.Vol. 62, No. 3, pp $119-130$.

[29] Serro R., Carreiro J.V, Galvao J.P and Peis R, (1992) Population dose assessment from radio-diagnosis in Portugal. Radiation Protection Dosimetry 43,65-68.

[30] Sharifat I , Olarinoye I and Oyeleke (2009) Patient Entrance skin doses at Minna and Ibadan for common diagnostic Radiological Examination, Bayero Journal of pure and applied sciences.2(1): 1-5

[31] Shrimpton D.C. Wall B.F, Jones D. G. Fisher E. S, Hiller, M. C. Kendall, G. M. and Harrison R. J. (2000) “A National Survey of Doses to Patients Undergoing a Selection of Routine X-ray Examination in English Hospitals"NRBP - 2000: Chilton, Decoct, Oxon.

[32] Shrimpton P. C, Wall B. F, and Jones D. G., A(1986) National Survey of Dose to Patients Undergoing a Selection of Routine X-ray Examination in English Hospitals NRPB - R 200. National RadiologicalProtection Board Oxen.

[33] Tung C. J, and Tsai H. Y,(1999) Evaluation of Gonad and fetal doses for diagnostic radiology, Proc. Nath. Sci. Council ROC (B), 1999, 23 (3): 107-113.

[34] UNSCEAR (1988).Sources and effects of Ionizing Radiation. United Nations Scientific Committee on theeffectsof Atomic Radiation, Report to General Assembly. Annex B exposure from Natural Radiation Sources. United Nations New York.

[35] UNSCEAR (1993) Sources and effects of Ionizing Radiation. United Nations Scientific Committee on the effects of Atomic Radiation, United Nations, New York.

[35] UNSCEAR (2000).Exposure from Natural Radiation Sources. United Nations Scientific Committee on theeffects of Atomic Radiation Report to General Assembly. Annex B exposure from Natural Radiation Sources. United Nations, New York.

[36] UNSCEAR, (2000).Sources and Effects of Ionizing Radiation. United Nations Scientific Committee on the Effects of Atomic Radiation, Report to the General Assembly. Annex B exposure from natural Radiation Sources. United Nations, New York.

[37] Wall B. F. and Shrimpton P. C. (1995) Patient dose Protocol and Tend in UK Radiation ProtectionDosimetry 57:359 - 362. 